\title{
Community-based Exercise Program for Solid Organ Transplant Recipients: Views of Exercise Professionals and Patients
}

\author{
Catherine Bednarczyk $^{1}$ | Catherine M. Tansey ${ }^{1}$ | Shawn Fontaine ${ }^{1}$ | \\ Stephanie Baker $^{1}$ | Érika Laberge ${ }^{1}$ | Sunita Mathur ${ }^{2,3}$ | Heather \\ Lambert $^{1}$ | Tania Janaudis-Ferreira ${ }^{1,3,4,5}$
}

\author{
${ }^{1}$ School of Physical and Occupational Therapy, Faculty of \\ Medicine, McGill University, Montreal Quebec \\ ${ }^{2}$ Department of Physical Therapy, University of Toronto, \\ Toronto, Canada \\ ${ }^{3}$ Canadian Donation and Transplantation Research \\ Program, Edmonton, Alberta, Canada \\ ${ }^{4}$ Translational Research in Respiratory Diseases Program, \\ Research Institute of the McGill University Health Centre, \\ Montreal, Canada \\ ${ }^{5}$ Centre for Outcomes Research and Evaluation (CORE), \\ Research Institute of the McGill University Health Centre, \\ Montreal, Canada

\section{Correspondence} \\ Tania Janaudis-Ferreira \\ Email: tania.janaudis-ferreira@mcgill.ca \\ Publication Date \\ January 30, 2021
}

\section{MJM 2021 (19) 5}

https://doi.org/10.26443/mjm.v19i1.218

\section{McGill Journal of Medicine}

www.mjmmed.com

\section{c) (1) (2)}

This work is licensed under a Creative Commons BY-NC-SA 4.0 International License.

\section{A BSTRACT}

Background: Community Based Exercise (CBE) programs have been shown to successfully increase exercise capacity and physical activity (PA) levels in different populations, however none exist for solid organ transplant (SOT) recipients.

Objective: To identify important factors when developing and implementing a CBE program for SOT recipients.

Methods: We conducted a qualitative study using semistructured interviews with seven SOT recipients and six exercise professionals (EPs). The data were analyzed using thematic analysis.

Results: Six themes were identified: 1) motivators to exercise: social benefits, improved quality of life and return to normal activities, and improved health; 2) perceived barriers to exercise: financial vulnerability post-transplantation, fear of injury, lack of exercise recommendations, and medication side effect; 3) level of supervision: recipients wanted guidance without overprotective supervision, while EPs were torn between extensive monitoring and promoting independence; 4) required education and foundational knowledge in EPs; 5) the importance of CBE programs for the SOT population: guidance and support to SOT recipients, with societal benefits; and 6) tailored program structure: group setting with individualized exercise prescription.

Conclusions: Recommendations may be used to develop an effective CBE program for SOT recipients, and thus improve PA levels among this population.

\section{KEYWORDS}

exercise, physical activity, community, transplant 


\section{1 | INTRODUCTION}

Solid Organ Transplant (SOT) is a life-saving procedure performed on individuals with organ failure. Despite substantial improvements in quality of life following the procedure, SOT recipients continue to have long-term limitations in exercise capacity (1) and low levels of physical activity (PA) may partly explain this. PA is defined as any bodily movement produced by the contraction of skeletal muscles that results in an increase in caloric requirements over resting energy expenditure. (2) A Canadian web-based questionnaire of 113 SOT recipients revealed that a large proportion of participants never engaged in light (60\%), moderate (55\%) or strenuous (43\%) recreational activities. (3) A study of women following heart transplantation demonstrated extremely low levels of PA with $55 \%$ of participants in the study reporting being inactive and only $15 \%$ engaged in moderate to high levels of PA. (4) Reduced PA and exercise capacity may increase SOT recipients' risk for developing secondary complications such as osteoporosis, diabetes, dyslipidemia, hypertension, and cardiovascular disease. $(5,6)$ These health complications may decrease transplant recipients' quality of life, while cardiovascular disease has been identified as the main cause of nontransplant-related death in this population. (7)

Exercise is defined as a type of PA that is planned, structured, and repetitive bodily movement done to improve and/or maintain one or more components of physical fitness. (2) Exercise training that includes aerobic and resistance exercises has been shown to improve exercise capacity, muscle strength, body composition, functional performance, mental health, and quality of life in the SOT population. $(5,8)$ In addition, exercise training has been shown to be effective in reducing risk factors for cardiovascular and metabolic disease in many chronic disease populations including SOT recipients. (9) To date, most published studies of exercise programs in SOT recipients were offered in an outpatient hospital setting. $(5,8)$ In Canada, most rehabilitation programs are also offered in a hospital setting, and a large disparity exists in regards to the provision of rehabilitation and exercise training services among organ types. (10) The majority of the programs are offered to heart and lung transplant candidates and recipients. (10) The most beneficial physical rehabilitation model would include preand post-transplant rehabilitation programs for all organ types; however, this type of program, if held in a hospital setting, has direct cost for the centre as it needs to hire qualified personnel and purchase equipment.

Community-based exercise (CBE) programs can be defined as a group of individuals with similar health conditions who perform exercise that is led by a physiotherapist or a fitness instructor. The goal of CBE is to promote exercise in a community setting by providing social support, supervision and guidance with exercise, (11) all of which have been identified as important facilitators to PA in the SOT population. (3) CBE programs have been successfully implemented and evaluated in individuals with stroke, cancer survivors, Alzheimer's disease, and older adults, while being safe, feasible, and effective in improving cognitive and physical function in these populations. (12)

To our knowledge, no specific CBE program exists for the SOT population. SOT recipients may face unique challenges related to higher risk of infections, side effects of immunosuppressive medications, (13) postsurgical complications, organ rejection, and fear of injury and losing the new organ. (14) Therefore, there is a need to understand the factors to be considered before developing a feasible, accessible, safe, and effective CBE program for SOT recipients.

The purpose of the present study was to identify important factors for the development and implementation of a CBE for stable SOT recipients in Canada, from the perspectives of key stakeholders such as SOT recipients and exercise professionals (EPs).

\section{2 | METHODS}

\section{1 | Study design}

We conducted a qualitative study using semi-structured interviews with SOT recipients and EPs with and without experience working with people with chronic diseases. Descriptive phenomenology, a method that in- 
vestigates the "pure" description and interpretation of people's experiences, (15) was used to understand the subjective reality of SOT recipients and EPs within their individual context. This study was approved by the ethics committee of McGill University (reference number A03-E11-18A), and all participants provided written informed consent. The reporting of this study follows the Consolidated Criteria for Reporting Qualitative Research. (16)

\section{2 | Study Participants}

\subsubsection{Individuals with SOT}

We recruited English speaking SOT recipients through The Canadian Network for Rehabilitation and Exercise for Solid Organ Transplant Optimal Recovery (CANRESTORE) mailing list and social media accounts (Facebook and Twitter) (a network about exercise and rehabilitation in transplantation; www.canrestore.ca). Purposive sampling was used to gain a variety of views, and participants were recruited from urban centers and their surrounding areas, which have major transplant centers. We aimed to recruit individuals with different organ transplants types (kidney, liver, heart, lung, etc.), stages of recovery (1-2 years, 2-5 years, 5+ years posttransplant), and gender. Only liver and heart recipients were found, and one participant from a rural area was included to get the perspective of a rural resident. Participants were excluded if less than one year had elapsed since their most recent transplantation. Twelve SOT recipients were contacted, five refused and seven completed the study.

\subsection{2 | EPs}

English speaking EPs were also recruited through the CAN-RESTORE mailing list, Facebook, and Twitter accounts. A minimum of two years of work experience was required from fitness and community centers in in the Montreal and Toronto area. EPs with and without experience with chronic disease or coordinating CBE programs for chronic conditions were included to provide a more heterogeneous sample and richer data. Ten EPs were contacted, three refused, and six completed the study.

\section{3 | Data Collection}

\subsection{1 | Demographics}

Age, gender, organ type, number of transplants received, date of last transplant, level of PA, and if current or previous participation in a CBE program were noted for all patient participants. The International PA Questionnaire Short Form (IPAQ-SF) assessed the level of PA. The 7item questionnaire is a valid and reliable instrument for measuring PA in individuals 15-69 years old. (17) Demographic data collected for EPs included age, gender, years of work experience, work setting, and population.

\subsection{2 | Semi-Structured Interviews}

Two investigators conducted in-depth, semi-structured interviews by telephone using a semi-structured interview guide. The interview guide was pilot tested with the first SOT recipient and EPs, and feedback was given on the clarity and type of questions. The pilot test interviews were included in data collection. Interviews were conducted from April to June 2018 and lasted 2045 minutes. The interview guide for the SOT participants contained eleven open-ended questions with additional probes relating to organ transplant experience, motivators and barriers to exercise, benefits and desired structure of a CBE program, and additional services that should be offered (Appendix). The interview guide for EPs included five open ended questions with additional probes and consisted of questions about their professional background and training, barriers in training and implementing programs for chronic disease, knowledge of and information they felt would be necessary to work with SOT recipients, and factors to consider in the development and implementation of a CBE for SOT (Appendix). All interviews were audio-recorded, transcribed verbatim, and reviewed by the interviewers to ensure accuracy. Recruitment was completed once it 
was deemed data saturation was reached. (18)

\section{4 | Data Analysis}

Interviews from SOT recipients and EPs were coded and analyzed using Thematic Analysis as described by Braun and Clark, (19) following a six-step procedure: 1) two researchers (EL, SF) developed preliminary codes; 2) transcripts were coded line-by-line without the use of software and initial codes were generated collaboratively; 3) codes were analyzed and then grouped into broader candidate themes; all themes were derived following the analysis of the data; 4) all extracted codes were reviewed and refined under each candidate theme to ensure a coherent pattern and fit of the thematic map to the data set. The entire data set was re-read and analyzed for missing or additional codes. 5) themes were named and defined; 6) final analysis of the data was completed and reported.

\section{3 |}

Thirteen participants took part in this study, including 3 (23\%) heart transplant recipients, 4 (31\%) liver transplant recipients, and 6 (46\%) EPs (Tables 1-2). Of the seven SOT participants, only one individual was attending a weekly post-transplant exercise program (hospitalbased). Six themes are described below.

\section{1 | Motivators to exercise}

\subsection{1 | Social benefits}

The most recurrent motivator to exercise was a group setting. All seven SOT recipients were enthusiastic about the psychosocial aspect of exercising with other transplant recipients. One participant exposed the sense of accountability that builds within a group that exercises together, and faces similar challenges:

"You got to talk to other people on how they were doing, [...], you're making a commitment to not just yourself, but to others as well." SOT6

\subsection{2 | Improved quality of life and return to normal activities}

The SOT recipients agreed that exercise experiences increase their perceived health-related quality of life and encourage a return to leisurely and meaningful activities.

"One of the things I wanted to do this year was to try and get conditioned back to be able to play a whole game [of hockey]." SOT4

\subsection{3 | Improved health}

Aware of the health risks that stem from deconditioning, three of the seven SOT recipients expressed health and weight loss as a motivation to exercise.

"I'm overweight. [...] I need to start taking care of my body and start exercising more." SOT3

\section{2 $\quad$ Perceived barriers to exercise}

\subsection{1 | General population barriers}

SOT recipients reported barriers to exercising (time constraints, cost, weather, physical accessibility, lack of motivation and fear of injury) identical to those commonly found within the general population. Furthermore, participants reinforced that these barriers are magnified by the process of receiving a new organ, and that transplant can lead to financial vulnerability.

"The cost of the program is a big thing, [...] we're not only managing a new transplant, but most of the people are managing maybe no job, or changes of jobs." SOT5 Indeed, the financial commitment of a gym membership is an expense that some could not have afforded immediately post-transplantation:

"As a recipient, until you get back on your feet financially, it's a struggle. I don't think I could afford it." SOT4

\subsection{2 | SOT recipient-specific barriers}

\subsubsection{1 | Fear}

It was a recurring theme that, post-transplant, SOT recipients were unsure how to exercise safely, due at least 
in part to having lived so long with a failing organ. SOT2 shared the additional initial fear of compromising the viability of her transplant:

"People don't know how much you can push yourself. You're always scared, your new heart is so precious, and you don't want to ruin this one." SOT2

\subsubsection{2 | Lack of physician's support}

SOT recipients expressed concerns about the lack of guidance about PA and precautions posttransplantation. SOT7 stated that he had to initiate the conversation with his physician or exercise would not have been mentioned. EP1 also expressed the consequences of a lack of exercise recommendations:

"When their doctor tells them something they'll listen, and if their doctor doesn't really talk about exercise [...], [patients] don't exercise." EP1

\subsubsection{3 | Medication side effects}

Participants reported a variety of medication side effects, and most recipients felt affected by medication. One recipient described how the side effects disrupted their quality of life:

"My biggest issue was prednisone and its side effects. It's painful and [...] I wouldn't be able to walk for a couple days due to muscle soreness." SOT5

All EPs agreed that medication side effects represent a major barrier to exercise intervention delivery.

"The [SOT] population is so heavily medicated, it's crazy. Anti-rejection medication interferes with their health, medical stability, and day-to-day physical state." EP1

\subsubsection{4 | Risks of infection}

Among SOT recipients, the management of infection risk was not a universal barrier. One claimed EPs should be aware of immune system fragility and incorporate preventive measures for spread of contagious illness in their exercise program. However, others did not consider the gym environment as an additional threat:

"I don't see the higher risk of getting an infection in a gym than going to the grocery store or getting in a subway. [...] Just wash your hands and be careful." SOT5

\section{3 | Level of supervision and medical clearance}

Among EPs and SOT recipients, the suggested amount of supervision varied. Three SOT recipients expressed that while they require guidance to exercise, trainers should not be overprotective, but simply attentive. Two SOT recipients expressed that despite having some unique differences when it comes to their ability to exercise, they wanted to be treated like normal people.

"Transplant recipients are not Martians or extraterrestrials." SOT5

"I think we can do pretty much anything, as long as the trainer doesn't overdo it." SOT3

On the other hand, SOT2, who spent 10 years in heart failure before receiving her transplant, reinforced the need of closely supervised programs as she was worried about her safety when she began exercising:

"often people with [a history of] heart failure get nervous to do too much, because for so long you couldn't."

EPs report a spectrum of supervision preference. For example, EP2 reinforced the need to initially assess all participants in order to provide the right amount of supervision and ensure safety. However, EP5, who runs a community-based program in partnership with a local hospital, thought each transplant recipient should be responsible for managing their condition once medically cleared:

"[Medical staff] are reluctant to allow individuals to be responsible for themselves. [But] rehabilitation teaches SOT recipients to know their level of exertion, how to pay attention to their body, and to be smart by making the decision not to come when they don't feel well." EP5

\subsection{Required education and foundational knowledge for EPs}

\subsection{1 | Medication and side effects}

Most EPs expressed the importance of knowing their clientele's medical background as well as their medications. The SOT recipients agreed that EPs need to be knowledgeable about the possible effects of their med- 
ications, and transplant process.

"Trainers should know how the body works once it's been transplanted, how the immune system works and what the immunosuppressive medications do." SOT6

\subsection{2 | General SOT knowledge}

SOT2 acknowledged that SOT recipients are underserved and that most EPs, including her kinesiologist, do not have the knowledge to treat them. She also highlighted the need for professional initiative and the effectiveness of learning through experience:

"When my kinesiologist started, he didn't have that specialized knowledge. [...] Over the years he actually increased his knowledge, not just on the physical impact of receiving a heart transplant, but also psychologically." SOT2

SOT4 not only preferred that his EPs have transplantspecific knowledge, but also stressed the need to be understood in his journey as a SOT recipient:

"I could go with anybody, but I would really feel comfortable if they have background knowledge and experience." SOT4

EPs were eager to learn about the nature of SOTs, and any specific exercise considerations. EP5 acknowledged the need for population-specific training. She also proposed helping to develop CBE programs through hospital partnerships:

"Fitness instructors who have undertaken general fitness training, they'll go to the hospital and observe therapeutic sessions of the program there. There they will get the population-specific training from the hospital, and they'll provide us with contraindications." EP5

Finally, EPs 1, 2, 3 and 4 expressed the need for evidence-based practices for exercise for SOT recipients.

\subsection{The importance of a CBE program: guidance and support to SOT recipients, with societal benefits}

All SOT recipients showed interest and willingness to participate in a CBE program. However, there is a lack of services and exercise guidance, especially for certain types of organ transplants:

"I see other transplant recipients like heart. Once they get out of the hospital, they have to follow a program. [...] I think it should be like that for all the transplant recipients." SOT3

CBE programs would also give SOT recipients peer support and an opportunity to acquire new knowledge about their condition.

"I've learned more about my side effects by training for and playing at the transplant games or from other transplant recipients, than any clinicians or pharmacists." SOT5

SOT5 stated that CBE programs should provide SOT recipients with adequate tools to manage their condition. He also exposed the societal benefits:

"The government would save a lot of money by sending someone to rehabilitation, knowing that they are sparing all the complications." SOT5

\section{6 | Tailored program structure with goal setting and progression monitoring}

\subsection{1 | Individualized exercise prescription}

Both SOT recipients and EPs made specific suggestions about how a CBE program for transplant recipients could be optimized (Table 3 ).

\section{4 | DISCUSSION}

To our knowledge, this is the first study to investigate essential factors for the development and implementation of a CBE program for the stable SOT population.

All participants reported that the CBE program should be conducted in a group setting with other SOT recipients, to provide social support such as sharing and learning from others, accountability, and a sense of normality and inclusion. Previous studies have found social support to be a large influential facilitator to being physically active in this population. (3) It was also found that group programs lead to greater exercise compliance (20) and intrinsic motivation to exercise (21) in healthy indi- 


\begin{tabular}{cccccc}
\hline SOT & Age & Gender & Organ & $\begin{array}{c}\text { Years } \\
\text { post-transplant }\end{array}$ & $\begin{array}{c}\text { IPAQ (hours spent in } \\
\text { moderate-vigorous } \\
\text { activities/week) }\end{array}$ \\
\hline 1 & 25 & Female & Liver & Hesire to be physically \\
active
\end{tabular}

IPAQ: International Physical Activity Questionnaire

CBE: Community Based Exercise

PA: Physical Activity

TABLE 1 SOT recipient characteristics $(n=7)$

\begin{tabular}{|c|c|c|c|c|}
\hline Participant & Gender & Profession & $\begin{array}{c}\text { Years of } \\
\text { experience }\end{array}$ & Location of Employment \\
\hline 1 & Female & Program manager/exercise leader & 25 & Community Center \\
\hline 2 & Female & Certified exercise physiologist & 12 & University affiliated Center \\
\hline 3 & Male & Kinesiologist & 10 & Private trainer \\
\hline 4 & Female & Certified exercise physiologist & 11 & University affiliated Center \\
\hline 5 & Female & Fitness Center Supervisor & 29 & Community Center \\
\hline 6 & Female & Community development coordinator of therapeutics & 13 & Community Center \\
\hline
\end{tabular}

TABLE 2 Characteristics of exercise professionals $(n=6)$

viduals. Most of the recipients participating in the study expressed a desire for a group to be composed solely of SOT recipients. EPs, who are more focused on feasibility, were generally more willing than SOT recipients to have mixed groups if fitness levels were matched. Although it is easier to integrate SOT recipients into already existing exercise classes, it would be more beneficial for this population to create a SOT only group. This reflects the findings of Estabrooks and Carron, who have shown that group cohesion, perception of similarity, and closeness within the group can predict shortand long-term adherence in older adult exercisers. (22)

SOT recipients and EPs also voiced different important features about the program. For recipients, having a program that offered some exercise prescription flexibility (e.g. group walks in the park) was key for participants to meet their individual goals. While EPs agreed, some were more focused on the program being in a context where recipients' vital signs can easily be monitored for safety. This might reflect the EPs' lack of experience of working with SOT recipients and therefore they feel hesitant not to monitor them.

The fact that EPs felt that a 12-week program would be an ideal duration for CBE programs, whereas SOT recipients would prefer a year-round program indicates that EPs are focused on short-term improvements in physical fitness and SOT recipients are focused on longterm physical fitness maintenance through regular PA. This highlights the importance of the development of PA programing rather than only short-term exercise-based programs for SOT recipients.

Previous studies reported that the cost of fitness centers and side effects post-transplant were major barriers to exercise for SOT recipients. $(14,23)$ Our participants also discussed these barriers, although recipients stressed the financial burden post-transplant and the importance of the program's location. Therefore, when implementing a successful CBE program, one needs to con- 


\begin{tabular}{|c|c|c|}
\hline & SOT Recipients & Exercise Professionals (EP) \\
\hline $\begin{array}{l}\text { Individual vs } \\
\text { group sessions }\end{array}$ & $\begin{array}{l}\text { Recipients wanted a balance between individual and } \\
\text { group exercise time. }\end{array}$ & $\begin{array}{l}\text { Some exercise professionals proposed starting with } \\
\text { individual sessions to set goals, and then be assigned } \\
\text { to an appropriate group. }\end{array}$ \\
\hline $\begin{array}{l}\text { Specific program } \\
\text { features }\end{array}$ & $\begin{array}{l}\text { The program should include transplant-specific } \\
\text { adaptations and exercise prescription flexibility, to } \\
\text { ensure everyone meets their goals. }\end{array}$ & $\begin{array}{l}\text { Some exercise professionals felt it was necessary to } \\
\text { monitor vital signs closely, while others felt it was okay } \\
\text { to rely on the patients' symptoms and judgement. }\end{array}$ \\
\hline $\begin{array}{l}\text { Group } \\
\text { Composition }\end{array}$ & $\begin{array}{l}\text { Mixed opinions: exercise with other patient population } \\
\text { groups vs exercise with SOT recipients only. The } \\
\text { majority preferred recipient only groups. }\end{array}$ & $\begin{array}{c}\text { No specific recommendation. Creating mixed } \\
\text { population, or SOT recipient specific groups based on } \\
\text { physical fitness were mentioned. }\end{array}$ \\
\hline Group Size & $\begin{array}{l}\text { Preferred } 10 \text { - } 20 \text { participants in the group, to ensure } \\
\text { adequate feedback and guidance. }\end{array}$ & $\begin{array}{l}\text { Mixed opinions on ratio size (exercise professionals: } \\
\text { SOT recipients). 2:16 for independent participants, and } \\
\text { as much as 1:2 for chronically ill patient groups. }\end{array}$ \\
\hline Accessibility & $\begin{array}{l}\text { The program should be accessible in terms of cost and } \\
\text { location. Target prices for a year-round membership } \\
\text { differed, ranging from having the first year free, to a } \\
\text { small cost. }\end{array}$ & No specific recommendation. \\
\hline Environment & Should be located somewhere in the neighborhood. & Same as SOT recipients. \\
\hline Duration & $\begin{array}{l}\text { 1-2 hours, } 2-3 x / \text { week, year-round to allow dropping in } \\
\text { and out based on fluctuating health status. }\end{array}$ & $\begin{array}{l}\text { 12-week program would be an ideal timeframe, due to } \\
\text { resource limitations and clinically meaningful health } \\
\text { outcome changes. }\end{array}$ \\
\hline Type & $\begin{array}{l}\text { Recipients were open to the type of exercise, liking a } \\
\text { mix of cardio and strength training, with the possibility } \\
\text { to make their choice in the form of exercise (for ex: } \\
\text { bike vs treadmill, etc). Group walks in a park were also } \\
\text { of interest, although only a viable option during the } \\
\text { warmer months, and not sufficient on its own due to } \\
\text { weather constraints. }\end{array}$ & $\begin{array}{l}\text { There was no consensus. Some felt outdoor group } \\
\text { walks were an option, due to the great social benefits. } \\
\text { Others didn't like the fact that vitals could not be } \\
\text { monitored and measured as easily as in a gym setting. }\end{array}$ \\
\hline
\end{tabular}

TABLE 3 SOT and exercise professionals' perspectives about factors in developing a CBE program

sider its cost and accessibility, so that recipients can participate despite financial hardship secondary to change in employment status and increased medical expenses.

Many recipients reported being fearful of exercising, concerned that they will injure themselves or jeopardize their new organ. Zelle et al. (14) also identified fear of movement as a barrier to exercise in kidney transplant recipients. This is partly why EPs stressed the importance of having physicians play a collaborative role. Provided there are no contra-indications, SOT recipients are reassured and can feel safer when exercising. Physician recommendations have been identified as an important facilitator to PA in SOT populations (3); therefore, increased physician advocacy on the benefits of life-long exercise are imperative. Once a physician endorses a CBE program for example, recipients could meet with an EPs for education and guidance to assuage their fears even more.

Another major concern of EPs was the lack of established evidence-based exercise guidelines. SOT recipients expressed that EPs should have a basic understanding of transplant procedures, common medication and side-effects, the recovery process, the psychological impact, and physiological response to exercise. Thus, educational resources and training should be developed for EPs so they better understand the SOT population and its unique concerns when it comes to safe exercise.

SOT recipients also expressed a desire for nutritional support to assist with weight management and for nutritional and lifestyle counselling. However, because recipients usually have access to a dietician at the hospital, it is perhaps not a priority in a CBE program. However, this expressed desire may indicate an increased need for nutrition referrals outside of a CBE program. 
Community-based programs would optimally partner with hospitals and rehabilitation centers in order to obtain medical clearance, collaborate with medical professionals, and to streamline the rehabilitative process. Adjunct services such as physiotherapy, psychology, social support, and nutrition were felt by our participants to be an asset to a newly developed CBE program. Offering these services would help recipients recover, and in turn, increase their adherence to exercise by increasing their confidence in their new life and preventing or managing injury. For financial reasons, strategic partnerships with medical facilities, transplant foundations, and municipal recreation organizations could be fostered to help improve sustainability for, affordability of, and access to the program for SOT recipients.

There are several limitations inherent to this study. Most participants live in urban areas and may not reflect the opinions and behavior of individuals in rural regions. Furthermore, no participating EPs had direct work experience with the SOT population; however to our knowledge, there is no CBE program specific to SOT recipients in Canada. The SOT recipients only included liver and heart transplant recipients, and therefore may not be generalized to other organ groups. Lastly, due to our recruitment strategy (CAN-RESTORE mailing list and social media accounts), the study has an overrepresentation of SOT recipients engaged in PA as indicated by the IPAQ scores and therefore the findings may not be generalizable to those recipients who are not physically active.

\section{5 | CONCLUSIONS}

This study gathered the opinions of key stakeholders that would be involved in a CBE program for stable SOT recipients and sets the stage for the development of a feasible and relevant program for this population. Future research should aim to look at the development, implementation, and evaluation of a CBE for SOT recipients.

\section{REFERENCES}

1. Williams TJ, McKenna MJ. Exercise limitation following transplantation. Compr Physiol. 2012;2(3):1937-79.

2. Caspersen CJ, Powell KE, Christenson GM. Physical Activity, Exercise, and Physical Fitness: Definitions and Distinctions for HealthRelated Research, Public Health Reports. Public Health Reports 1985;100(2):126-31.

3. Gustaw T, Schoo E, Barbalinardo C, et al. Physical activity in solid organ transplant recipients: Participation, predictors, barriers, and facilitators. Clin Transplant. 2017;31(4).

4. Evangelista LS, Dracup K, Doering L, Moser DK, Kobashigawa J. Physical activity patterns in heart transplant women. J Cardiovasc Nurs. 2005;20(5):334-9.

5. Didsbury M, McGee RG, Tong A, et al. Exercise training in solid organ transplant recipients: a systematic review and meta-analysis. Transplantation. 2013;95(5):679-87.

6. Laish I, Braun M, Mor E, et al. Metabolic syndrome in liver transplant recipients: prevalence, risk factors, and association with cardiovascular events. Liver Transpl. 2011;17(1):15-22.

7. Pilmore H, Dent H, Chang S, McDonald SP, Chadban SJ. Reduction in cardiovascular death after kidney transplantation. Transplantation. 2010;89(7):851-7.

8. Janaudis-Ferreira T, Mathur S, Deliva R, et al. Exercise for Solid Organ Transplant Candidates and Recipients: A Joint Position Statement of the Canadian Society of Transplantation and CANRESTORE. Transplantation. 2019;103(9):e220-e38.

9. Li C, Xu J, Qin W, Hu Y, Lu H. Meta-Analysis of the Effects of Exercise Training on Markers of Metabolic Syndrome in Solid Organ Transplant Recipients. Progress in transplantation (Aliso Viejo, Calif). 2018;28:278-87.

10. Trojetto T, Elliott RJ, Rashid S, et al. Availability, characteristics, and barriers of rehabilitation programs in organ transplant populations across Canada. Clinical Transplantation. 2011;25.

11. Stuart M, Benvenuti F, Macko R, et al. Community-based adaptive physical activity program for chronic stroke: feasibility, safety, and efficacy of the Empoli model. Neurorehabil Neural Repair. 2009;23(7):726-34.

12. Carter ND, Khan KM, McKay HA, et al. Community-based exercise program reduces risk factors for falls in 65- to 75-yearold women with osteoporosis: randomized controlled trial. CMAJ. 2002;167(9):997-1004.

13. Fishman JA. Infection in solid-organ transplant recipients. $\mathrm{N}$ Engl J Med. 2007;357(25):2601-14.

14. Zelle DM, Corpeleijn E, Klaassen G, et al. Fear of Movement and Low Self-Efficacy Are Important Barriers in Physical Activity after Renal Transplantation. PLoS One. 2016;11(2):e0147609.

15. Matua GA, Van Der Wal DM. Differentiating between descriptive and interpretive phenomenological research approaches. Nurse Res. 2015;22(6):22-7.

16. Tong A, Sainsbury P, Craig J. Consolidated criteria for reporting qualitative research (COREQ): a 32 -item checklist for interviews 
and focus groups. Int J Qual Health Care. 2007;19(6):349-57.

17. Lee PH, Macfarlane DJ, Lam TH, Stewart SM. Validity of the International Physical Activity Questionnaire Short Form (IPAQ-SF): a systematic review. Int J Behav Nutr Phys Act. 2011;8:115.

18. Guest G, Bunce A, Johnson L. How many interviews are enough? An experiment with data saturation and variability. Field methods. 2006;18(1):59-82.

19. Braun V, Clarke V, Hayfield N, Terry G. Thematic analysis. Handbook of Research Methods in Health Social Sciences. 2019:843-60. 20. Fraser SN, Spink KS. Examining the role of social support and group cohesion in exercise compliance. J Behav Med. 2002;25(3):233-49.

21. McNeill LH, Wyrwich KW, Brownson RC, Clark EM, Kreuter MW. Individual, social environmental, and physical environmental influences on physical activity among black and white adults: a structural equation analysis. Ann Behav Med. 2006;31(1):36-44.

22. Estabrooks PA, Carron AV. Group cohesion in older adult exercisers: prediction and intervention effects. J Behav Med. 1999;22(6):575-88.

23. van Adrichem EJ, van de Zande SC, Dekker R, et al. Perceived Barriers to and Facilitators of Physical Activity in Recipients of Solid Organ Transplantation, a Qualitative Study. PLOS ONE. 2016. 


\section{Appendix: Interview Guide}

\section{1 | SOT Recipient}

Can you start by telling me about your transplant experience and what type of organ transplant you received?

- When was your transplant?

- Did you experience any complications?

How would you describe your current level of physical activity?

- What type of activities or exercise do you currently do and how often?

- Were you physically active before your transplant? If so, what type of exercise did you do and how often?

What motivates you to exercise? What are your goals?

- What type of exercise or activities do you like best?

What barriers do you face when it comes to doing exercise?

- Are any of these barriers related to your organ transplant?

Do you think a community exercise program would be beneficial for solid organ transplant recipients? Why? Why not?

Note: A community exercise program is an activity involving exercise, that is offered to a specific population (in this case SOT recipients) and led by a trained fitness instructor.

What would encourage you to participate in a community-based exercise program?

What would discourage you from participating in a community-based exercise program?

Do you prefer group training or one-on-one training (with a trainer)?

How do you think a community-based exercise program for transplant recipients should look like?

- What should be considered when it comes to the types of classes offered, length (30min, location, expertise of the instructors, group size, membership cost, accessibility, etc.?

- Would you prefer to exercise outdoors in a park (e.g. walking group, yoga in a park, etc)?

- What do you think an exercise trainer needs to know about transplant to help you with your exercise program (for instance, do they need to know about medications (immunosuppressants), risk of infections, etc)?

Are there any additional services you think should be offered in a community-based exercise program besides exercise (nutritional support, psychological support, etc.)?

Is there a location in your community that you would go to exercise (with other transplant recipients)?

\section{2 | Exercise Professionals}

What is your professional background and training? 
- How long have you been working in the field of exercise training?

- How does your job relate to implementing exercise prescription or programming in the community?

- What chronic disease populations do you work with?

- Can you describe the program you run (number of participants, how often it is given, types of exercises, type of supervision)?

What barriers or challenges do you face when implementing exercise to people with chronic diseases?

What barriers do you think people with chronic diseases face when it comes to exercising or adhering to an exercise program?

Have you ever worked with solid organ transplant recipients before?

- Would you feel comfortable working with solid organ transplant recipients?

- What type of information do you feel you would need, in order to be confident working with this population?

- What challenges do you foresee with regards to implementing an exercise program for this population?

- Would you feel comfortable working with a recently transplanted recipient?

If your center decided to create a community exercise program for solid organ transplant recipients, how would it look like?

- Would individual or group sessions be recommended?

- Would the center be able to offer this population a reduced rate?

- Would activities outdoor (walking program, Yoga at the Park) be an option? What would be the advantages and disadvantages of such programs? 